\title{
AVALIAÇÃO DO USO DE INIBIDORES DE ETILENO SOBRE A PRODUÇÃO DE COMPOSTOS VOLÁTEIS E DE MANGIFERINA EM MANGA
}

\author{
Kirley Marques Canuto*, Manoel Alves de Souza Neto e Deborah dos Santos Garruti \\ Embrapa Agroindústria Tropical, CP 3761, 60511-110 Fortaleza - CE, Brasil \\ Maria Auxiliadora Coêlho de Lima \\ Embrapa Semiárido, CP 23, 56302-970 Petrolina - PE, Brasil
}

Recebido em 5/1/10; aceito em 13/3/10; publicado na web em 29/6/10

\begin{abstract}
EVALUATION OF THE USE OF ETHYLENE INHIBITORS ON PRODUCTION OF VOLATILE COMPOUNDS AND MANGIFERIN IN MANGO FRUIT. Effects of two ethylene inhibitors, 1-methylcylopropene (1-MCP) and aminoethoxyvinylglycine (AVG), on production of volatile compounds and mangiferin (a bioactive xanthone) in 'Tommy Atkins' mango fruit were investigated. Volatile composition and mangiferin content, in treated and untreated fruits at three maturity, stages were determined by SPME-GC-MS and HPLC, respectively. These chromatographical analysis revealed that the volatile profiles and mangiferin concentrations were not significantly different, suggesting that the use of ethylene inhibitors does not affect the mango aroma and functional properties relative to this xanthone. Moreover, a simple, precise and accurate HPLC method was developed for quantifying mangiferin in mango pulp.
\end{abstract}

Keywords: Mangifera indica; ethylene inhibitors; mangiferin.

\section{INTRODUÇÃO}

Inibidores de etileno são agentes químicos capazes de inibir ou retardar os efeitos do etileno, um fito-hormônio ubíquo nos vegetais superiores e responsável por regular várias respostas fisiológicas. ${ }^{1}$ Em virtude do papel importante do etileno na indução do amadurecimento de frutos climatéricos, inibidores de etileno tais como 1-metil-ciclopropeno (1-MCP) e aminoetoxi-vinil-glicina (AVG) têm sido empregados com sucesso na conservação pós-colheita de frutas e hortaliças. ${ }^{1,2}$

Em frutas como maçã, abacate, pera e manga, o efeito dos inibidores de etileno no retardo do amadurecimento pode ser facilmente evidenciado pelo atraso na mudança de coloração da casca/polpa e pela maior firmeza da polpa em relação a frutos não tratados. $\mathrm{O}$ efeito observado depende do tipo de inibidor utilizado (dose, $\mathrm{n}^{\circ}$ de aplicações, duração e temperatura do tratamento) e da fruta tratada (cultivar e estádio de maturação na colheita).,

$\mathrm{O}$ uso de inibidores de etileno propicia um incremento na vida útil de frutas, que pode viabilizar a comercialização para mercados mais distantes dos países produtores. Entretanto, aplicações de inibidores de etileno podem promover efeitos indesejáveis que geram dúvidas quanto aos seus benefícios, como alterações no aroma da fruta, e efeitos deletérios sobre a presença de compostos de importância nutricional, ${ }^{2}$ repercutindo negativamente na aceitabilidade da fruta pelo consumidor e, consequentemente, no valor de mercado do produto. ${ }^{4}$

AVG e 1-MCP afetaram a produção total de compostos voláteis (terpenos, cetonas, aldeídos, ésteres e alcoóis de baixo peso molecular) em banana, maçã, pera, melão e manga, sendo que alguns deles são componentes odoríferos característicos do aroma e do sabor destas frutas. ${ }^{5-10}$ Em morango, pera, abacaxi e manga, inibidores de etileno também afetaram os teores de substâncias bioativas (ex.: vitamina $\mathrm{C}$ e flavonoides), ${ }^{2,11,12}$ as quais são hoje amplamente valorizadas pela população devido às suas propriedades benéficas à saúde. ${ }^{4}$

Em manga 'Tommy Atkins' (variedade mais cultivada no Brasil), ${ }^{13}$ a aplicação de inibidores de etileno prolongou em alguns dias a vida útil dos frutos, através do retardo no amaciamento e na mudança da

*e-mail: kirley@cnpat.embrapa.br coloração da polpa, bem como pela limitação da perda de massa. ${ }^{14}$ Entretanto, não há informações a respeito do efeito destes agentes sobre o aroma e o teor de substâncias bioativas. $\mathrm{O}$ aroma desta cultivar é composto essencialmente por monoterpenos (70-90\%), principalmente $\delta$-3-careno, $\alpha$-pineno e $\alpha$-terpinoleno. ${ }^{15}$ As propriedades funcionais da manga podem ser atribuídas em grande parte à mangiferina (Figura 1), uma xantona C-glicosilada, encontrada em toda a planta, sendo mais abundante nas folhas e na casca do caule. ${ }^{16}$ Ensaios farmacológicos in vitro e in vivo mostraram que a mangiferina apresenta atividades antioxidante, anti-inflamatória, hipoglicemiante, hipolipidêmica, anticâncer, gastroprotetora, entre outras. Em razão do seu reconhecido potencial biológico, formulações contendo mangiferina vêm sendo exploradas comercialmente com fins nutricionais (suplemento alimentar) e farmacêuticos (fitoterápicos e cosméticos). ${ }^{17}$<smiles></smiles>

Figura 1. Estrutura química de mangiferina

O presente trabalho visou investigar a influência dos inibidores de etileno 1-MCP e AVG sobre a produção de mangiferina e de compostos voláteis na manga 'Tommy Atkins', mediante avaliação comparativa do perfil de voláteis e dos teores de mangiferina em mangas tratadas e não tratadas.

\section{PARTE EXPERIMENTAL}

\section{Materiais}

Solventes: metanol grau HPLC (MeOH, Tedia Co, Fairfield, EUA), água purificada (18 $\mathrm{m} \Omega$ ) por sistema Milli-Q (Millipore, Bedford, USA), $n$-butanol grau analítico ( $n$-BuOH, Synth, Diadema, Brasil). Fibra de SPME (microextração de fase sólida) de sílica fundida 50/30 $\mu \mathrm{m}$ divinilbenzeno/carboxen/polidimetilsiloxano, da Supelco (Bellefonte, EUA). Cloreto de sódio $(\mathrm{NaCl})$ e sulfato de sódio 
anidro ( $\mathrm{Na}_{2} \mathrm{SO}_{4}$, Vetec, Rio de Janeiro, Brasil), ácido trifluoroacético $\left(\mathrm{CF}_{3} \mathrm{CO}_{2} \mathrm{H}\right.$, Tedia $\mathrm{Co}$, Fairfield, EUA). Inibidores de etileno: AVG Retain $^{\mathrm{TM}}\left(\mathrm{C}_{6} \mathrm{H}_{12} \mathrm{~N}_{2} \mathrm{O}_{3}\right.$, Sumitomo Chemical, Osaka, Japão, 15\% i.a.) e 1-MCP SmartFresh ${ }^{\mathrm{TM}}\left(\mathrm{C}_{4} \mathrm{H}_{6}\right.$, Agrofresh Inc., Springhouse, EUA, 0,14\% i.a.). Padrão: mangiferina $\left(\mathrm{C}_{19} \mathrm{H}_{18} \mathrm{O}_{11}\right.$, Sigma Aldrich, St. Louis, EUA, 99\%).

\section{Equipamentos}

As análises por cromatografia gasosa-espectrometria de massas (CG-EM) foram realizadas em um instrumento Shimadzu QP-2010 (Kioto, Japão), com impacto de elétrons a 70 eV, coluna DB-5MS metilpolissiloxano (30 m x 0,25 mm x 1,0 $\mu \mathrm{m}$; J\&W Scientific Inc., Folsom, EUA). As análises por cromatografia líquida de alta eficiência (CLAE) foram desenvolvidas em um cromatógrafo Waters-1525 (Milford, EUA) composto por um sistema de bomba binária, injetor manual Rheodyne $($ loop $=20 \mu \mathrm{L})$, coluna XTerra RP-18 (4,6 x 250 $\mathrm{mm}, 5 \mu \mathrm{m}$ ), mantida em forno termostático a $35^{\circ} \mathrm{C}$, acoplado a um detector de arranjo de fotodiodo Waters-2996.

\section{Frutos}

Mangas 'Tommy Atkins' (Mangifera indica L.) colhidas no estádio de maturação 2 (caracterizadas pelo formato típico da cultivar, pela coloração da casca mudando para verde claro e textura ainda firme), fisicamente uniformes e visualmente livres de doenças, foram adquiridas na empresa Copa Fruit Importação e Exportação Ltda, localizada em Petrolina, Pernambuco (Brasil), em agosto de 2008/2009. Os frutos foram lavados com água corrente e mantidos à temperatura ambiente. O estudo foi realizado com frutos nos estádios de maturação 2 (ponto de colheita - EM2), 3 (casca com coloração verde amarelado e início de perda de firmeza - EM3) e 4 (frutos com casca amarela, macios e, por conseguinte, maduros - EM4).

\section{Tratamento com inibidores de etileno}

Os inibidores de etileno foram aplicados em frutos intactos, um dia após a colheita, seguindo-se as recomendações de preparo de solução fornecidas pelos fabricantes destes produtos. No tratamento com 1-MCP, os frutos foram acondicionados em uma caixa plástica hermeticamente fechada $\left(0,186 \mathrm{~m}^{3}\right)$, onde foram expostos a uma dose de $1200 \mathrm{~nL} \mathrm{~L}^{-1}$ do inibidor por $18 \mathrm{~h}$. A aplicação de AVG foi realizada mediante imersão dos frutos em um banho contendo $200 \mu \mathrm{g} \mathrm{g}^{-1}$, durante 2 min. Frutos que não foram submetidos a qualquer tratamento compuseram o grupo controle, sendo, como os demais, conservados à temperatura ambiente. As doses aplicadas foram definidas a partir de estudos prévios, os quais revelaram as concentrações mais eficientes em retardar o amadurecimento de manga 'Tommy Atkins' ${ }^{14}$

\section{Preparo e análise das amostras}

As amostras foram preparadas nos períodos correspondentes a cada estádio de maturação, ou seja, à medida que os frutos amadureciam. Os frutos tratados e não tratados foram cortados longitudinalmente e divididos em vários pedaços, utilizando-se faca de aço inoxidável. Nas análises cromatográficas em fases gasosa e líquida foram empregados, respectivamente, dois e três frutos para cada tratamento.

\section{Componentes voláteis}

Os componentes voláteis da polpa de manga foram extraídos por SPME e analisados por CG-EM, segundo metodologia descrita por Canuto e colaboradores. ${ }^{15} \mathrm{Na}$ extração, foram utilizadas porções de
$44 \mathrm{~g}$, as quais foram homogeneizadas em solução saturada de $\mathrm{NaCl}$ (100 mL), utilizando-se microprocessador doméstico. Alíquotas de $8 \mathrm{~mL}$ de cada suco produzido foram imediatamente transferidas para frascos de $40 \mathrm{~mL}$ e hermeticamente fechadas com tampas rosqueáveis, contendo septo de silicone. A identificação dos compostos foi realizada pela análise dos padrões de fragmentação exibidos nos espectros de massas, tendo sido confirmada por comparação dos seus espectros de massas com aqueles presentes na base de dados fornecida pelo equipamento (NIST- 147.198 compostos), bem como através da comparação dos seus índices de retenção com os de compostos conhecidos, obtidos por injeção de uma mistura de padrões contendo uma série homóloga de alcanos $\mathrm{C}_{7}-\mathrm{C}_{22}$, e dados da literatura. ${ }^{18}$

\section{Mangiferina}

Os teores de mangiferina foram determinados por CLAE, empregando-se método desenvolvido e validado neste estudo. As amostras foram preparadas a partir de porções de polpa fatiada (60 g) e homogeneizadas com água destilada $(175 \mathrm{~mL})$. O suco resultante foi filtrado a vácuo e particionado com $n$ - $\mathrm{BuOH}(3 \times 80 \mathrm{~mL})$. A fração $n$-butanólica foi tratada com $\mathrm{Na}_{2} \mathrm{SO}_{4}$ anidro, posteriormente filtrada e seca em evaporador rotativo $\left(60^{\circ} \mathrm{C}\right)$.

\section{Validação do método de cromatografia líquida de alta eficiência}

\section{Condições cromatográficas}

O método cromatográfico consistiu de uma corrida isocrática, empregando-se solução aquosa de $\mathrm{CF}_{3} \mathrm{CO}_{2} \mathrm{H} 1 \% / \mathrm{MeOH}$ (65:35) como fase móvel, durante $10 \mathrm{~min}$ e fluxo de $1 \mathrm{~mL} / \mathrm{min}$. O volume de injeção foi de $20 \mu \mathrm{L}$ e a detecção por ultravioleta foi registrada a $258 \mathrm{~nm}(\lambda)$.

\section{Preparo das soluções: estoque, padrão, controle de qualidade e amostra}

A solução estoque foi preparada, em triplicata, pela dissolução de $10 \mathrm{mg}$ de mangiferina (substância de referência) em $10 \mathrm{~mL}$ de solução aquosa de $\mathrm{CF}_{3} \mathrm{CO}_{2} \mathrm{H} 1 \% / \mathrm{MeOH}(1: 1)$. Retirando-se alíquotas apropriadas da solução estoque, foram obtidas 6 soluções padrão de mangiferina de $10 \mathrm{~mL}(300,200,100,50,10$ e $5 \mu \mathrm{g} / \mathrm{mL})$. Três soluções de controle de qualidade de mangiferina (250, 130 e 20 $\mu \mathrm{g} / \mathrm{mL}$ ) foram produzidas através de diluições sucessivas, a partir da solução padrão mais concentrada $(300 \mu \mathrm{g} / \mathrm{mL})$. Os volumes de todas as soluções foram complementados com porções da fase móvel. As soluções amostra foram preparadas pela dissolução de $20 \mathrm{mg}$ da fração $n$-butanólica, obtida por partição líquido-líquido do suco de manga (produzido com frutos nos três diferentes estádios de maturação), em $1 \mathrm{~mL}$ de solução aquosa de $\mathrm{CF}_{3} \mathrm{CO}_{2} \mathrm{H} 1 \% / \mathrm{MeOH}$ (1:1). As soluções estoque e amostra foram filtradas através de membranas de teflon $(0,45 \mu \mathrm{m}$, Waters $)$.

\section{Curva de calibração}

As curvas de calibração de mangiferina foram construídas em duplicata, em 3 dias não consecutivos, a partir das áreas dos picos obtidas pela injeção das 6 soluções padrão. A linearidade das curvas analíticas foi avaliada através da determinação da equação da reta e do coeficiente de correlação linear.

\section{Precisão}

A precisão do método foi examinada por comparação das concentrações obtidas para soluções de controle de qualidade de mangiferina em níveis baixo $(20 \mu \mathrm{g} / \mathrm{mL})$, médio $(130 \mu \mathrm{g} / \mathrm{mL})$ e alto $(250 \mu \mathrm{g} / \mathrm{mL})$, injetadas em triplicata. A repetitividade foi determinada ao longo de um dia e durante 3 dias não consecutivos, sendo expressa como coeficiente de variação (CV \%). 


\section{Exatidão}

A exatidão do método foi mensurada através da comparação das concentrações de mangiferina determinadas através da curva de calibração (valores experimentais) em relação às concentrações das soluções de controle de qualidade (valores de referência), nos três níveis (baixo - $20 \mu \mathrm{g} / \mathrm{mL}$, médio - $130 \mu \mathrm{g} / \mathrm{mL}$ e alto - $250 \mu \mathrm{g} / \mathrm{mL}$ ). As soluções foram analisadas em triplicata.

\section{Análise estatística}

Os resultados foram estatisticamente analisados pelo programa Microsoft Office ${ }^{\circledR}$ Excel 2003, através da análise de variância (ANOVA), comparando-se as médias pelo teste de Tukey, com $\mathrm{P} \leq 0,05$.

\section{RESULTADOS E DISCUSSÃO}

Análises de CG-EM demonstraram que, de modo geral, os inibidores de etileno 1-MCP e AVG não afetam a composição volátil da manga, uma vez que não foi observada diferença significativa nas áreas relativas dos picos dos componentes presentes em frutos tratados e não tratados, em qualquer um dos estádios de maturação avaliados (Tabela 1). Considerando-se o conjunto total de amostras, foram detectadas 48 substâncias e identificadas 43 . O aroma foi constituído basicamente por monoterpenos $(73,30-93,02 \%)$, sendo $\delta$-3-careno $(25,93-60,80 \%)$ o composto majoritário. Os dois inibidores de etileno utilizados também não foram capazes de interferir no processo natural de formação dos principais compostos voláteis. Assim, ao longo do amadurecimento dos frutos, houve queda acentuada na proporção de $\delta$-3-careno, incremento na produção de sesquiterpenos (principalmente trans- $\beta$-cariofileno) e de alguns monoterpenos ( $\alpha$-pineno e $\alpha$-terpinoleno), bem como síntese de ésteres apenas na manga madura (estádio de maturação 4), tal como observado previamente em estudo sobre as mudanças na composição volátil de manga 'Tommy Atkins' durante a maturação. ${ }^{15}$

Contudo, a aplicação de inibidores de etileno provocou diferenças sutis no número e no tipo de compostos, bem como influenciou o período de síntese de alguns constituintes cuja presença está intrinsecamente associada a um estádio de maturação.

Em todos os estádios de maturação, a maior quantidade de componentes voláteis foi detectada em frutos tratados com 1-MCP: 25 (EM2), 33 (EM3) e 29 compostos (EM4), contra 22/22 (EM2), 25/26 (EM3) e 27 (EM4) compostos registrados em frutos tratados com AVG e no grupo controle, respectivamente. A exposição dos frutos a inibidores de etileno levou ainda ao desenvolvimento de certas substâncias que não constam na composição original de manga 'Tommy Atkins': p-menta-1,5-dien-8-ol, isomentol, $(E)$-anetol (1-MCP), $\delta$-2-careno, butanoato de isoamila e butanoato de butila (AVG e 1-MCP). Porém, $\gamma$-elemeno, $\alpha$-amorfeno e octanoato de etila foram característicos dos frutos isentos de inibidores de etileno.

Quanto aos compostos responsáveis por notas verdes do aroma, o efeito dos inibidores de etileno foi antagônico. Enquanto AVG suprimiu o conteúdo de $\beta$-ocimeno e $\beta$-felandreno, na manga madura, 1-MCP estendeu o período de síntese de $\beta$-tujeno (EM4- 0,06\%) e eucarvona (EM3- 0,09\%). Em maçãs, frutos submetidos a tratamento com 1-MCP exibiram uma maior quantidade de componentes remanescentes do estádio pré-climatérico (hexanol, 2-hexenal e $\beta$-damascenona), apresentando maior aceitação em uma avaliação sensorial do que frutos não tratados. ${ }^{7}$

Inibidores de etileno induziram a síntese precoce de etanol, uma substância típica da maturação dos frutos desta variedade. ${ }^{15}$ Desta forma, a presença de etanol foi verificada já no estádio inicial em frutos tratados com 1-MCP (EM2- 0,69\%; EM3- 0,76\% e EM4$0,66 \%$ ) e a partir do período intermediário de maturação para aqueles submetidos a tratamento com AVG (EM3-1,53\%; EM4- 3,43\%). A formação de canfeno (EM2- 0,06\%) foi igualmente antecipada por ação do 1-MCP. Por outro lado, AVG atrasou o aparecimento de $p$ cimen-8-ol (EM4- 0,20\%) e prolongou a produção de limonen-4-ol (EM4- 0,18\%)

Na manga madura, o uso de inibidores de etileno favoreceu a geração de uma maior diversidade de ésteres, os quais são substâncias de grande importância odorífera para manga. Seis compostos foram identificados em frutos contendo AVG, 5 na manga exposta a 1-MCP e 4 no grupo controle. No entanto, metacrilato de etila e butanoato de etila (o componente de maior impacto no aroma de 'Tommy Atkins') ${ }^{15}$ mantiveram-se como os ésteres mais abundantes.

Inversamente, 1-MCP e AVG inibiram a produção generalizada de compostos voláteis (ésteres, aldeídos, alcoóis, cetonas e terpenos) em maçã, pera, melão, banana e manga 'Kensigton Pride', causando forte impacto no aroma ${ }^{6-10} \mathrm{Em}$ testes sensoriais, a preferência do consumidor pelas maçãs 'Anna' e 'Gala' mostrou-se controversa, enquanto que a aceitação de peras 'Conference' não foi afetada. ${ }^{7,8}$ O mecanismo de ação pelo qual inibidores de etileno influenciam a biossíntese de compostos voláteis ainda é desconhecido, porém se acredita que algumas enzimas responsáveis pela formação de compostos odoríferos sejam inibidas. ${ }^{19}$ Em melões e bananas 'Williams', a queda na produção de ésteres e concomitante elevação no conteúdo total de alcoóis sugeriram inibição da enzima álcool-aciltransferase. ${ }^{9}$ Em damascos, o crescimento na síntese de terpenoides foi justificado pela ativação da ação enzimática da $\alpha$-glicosidase, que liberou os terpenos ligados a açúcares da matriz. ${ }^{20}$ Em manga, o declínio na produção de componentes do aroma foi atribuído à diminuição na síntese de ácidos graxos, os quais são precursores de várias classes de compostos voláteis. ${ }^{10}$

Em relação à investigação sobre o efeito dos inibidores de etileno na produção de mangiferina, foi desenvolvido um método seletivo, exato e reprodutivo para a determinação dos teores desta xantona glicosilada em manga 'Tommy Atkins', utilizando-se CLAE. O método cromatográfico foi estabelecido em coluna de fase reversa, utilizando-se solução aquosa de $\mathrm{CF}_{3} \mathrm{CO}_{2} \mathrm{H} 1 \% / \mathrm{MeOH}$ (65:35, modo isocrático) como fase móvel e detecção por arranjo de fotodiodo. O método proporcionou uma boa separação entre a substância de interesse e os demais constituintes da polpa de manga, em um tempo adequado (tempo de corrida $=10 \mathrm{~min}$ ). Varredura espectral na região do ultravioleta confirmou a pureza do pico e permitiu selecionar o melhor comprimento de onda para visualização do cromatograma $\left(\lambda_{\text {máx }}=258 \mathrm{~nm}\right)$.

As curvas de calibração analítica foram construídas a partir de 6 níveis de concentração de mangiferina e devidamente ajustadas por regressão linear. As equações de regressão e os coeficientes de correlação (Tabela 2) revelaram relações de proporcionalidade direta entre as concentrações e as áreas do pico. A linearidade foi compatível com os valores de $\mathrm{r}$ recomendados pela ANVISA (> 0,99). ${ }^{21}$ A precisão foi mensurada pelo grau de repetitividade intradia e interdia dos ensaios, ou seja, por análises repetidas ao longo de um dia e realizadas em 3 dias não consecutivos, respectivamente. Este parâmetro foi determinado através do coeficiente de variação (CV) das áreas do pico de mangiferina, produzidas pela injeção de soluções de controle de qualidade em 3 níveis de concentração (20, 130 e $250 \mu \mathrm{g} / \mathrm{mL}$ ). As determinações mais precisas foram obtidas nas análises intradia $(>0,20 \%)$ e interdia $(>1,32 \%)$ das soluções mais concentradas. Excetuando-se a análise da solução de concentração intermediária, realizada no $2^{\circ}$ dia de experimento, todas as medições apresentaram graus de concordância inferiores a $5 \%$ (limite sugerido pela ANVISA), evidenciando a reprodutibilidade do método. A exatidão foi aferida através da comparação entre as concentrações determinadas experimentalmente $\left(\mathrm{C}_{\mathrm{e}}\right)$ e os valores de concentração 
Tabela 1. Composição química volátil de manga 'Tommy Atkins', tratada com inibidores de etileno, em três estádios de maturação

\begin{tabular}{|c|c|c|c|c|c|c|c|c|c|c|}
\hline \multirow{2}{*}{ Componente } & \multirow{2}{*}{ IR } & \multicolumn{3}{|c|}{ Estádio de Maturação $2^{a}$} & \multicolumn{3}{|c|}{ Estádio de Maturação $3^{\mathrm{a}}$} & \multicolumn{3}{|c|}{ Estádio de Maturação $4^{\mathrm{a}}$} \\
\hline & & AVG & 1-MCP & Controle & AVG & 1-MCP & Controle & AVG & 1-MCP & Controle \\
\hline não identificado 1 & - & $2,25 \pm 0,22 \mathrm{a}$ & $2,13 \pm 0,39 a$ & $1,94 \pm 0,23 a$ & $1,02 \pm 0,63 \mathrm{a}$ & $1,08 \pm 0,83 \mathrm{a}$ & $1,01 \pm 0,16 \mathrm{a}$ & $1,42 \pm 0,25 \mathrm{a}$ & $1,06 \pm 0,37 \mathrm{a}$ & $0,91 \pm 0,05 \mathrm{a}$ \\
\hline não identificado 2 & - & $0,19 \pm 0,11 \mathrm{a}$ & $0,73 \pm 0,87 \mathrm{a}$ & $0,34 \pm 0,10 \mathrm{a}$ & $0,51 \pm 0,15 \mathrm{a}$ & $0,6 \pm 0,85$ & - & $0,92 \pm 0,21 \mathrm{a}$ & $0,25 \pm 0,35 \mathrm{a}$ & $2,29 \pm 2,31 \mathrm{a}$ \\
\hline etanol & - & - & $0,69 \pm 0,98$ & - & $1,53 \pm 0,40$ & $0,76 \pm 0,17$ & - & $3,43 \pm 0,02 \mathrm{a}$ & $0,66 \pm 0,01 \mathrm{a}$ & $0,82 \pm 1,60 \mathrm{a}$ \\
\hline não identificado 3 & - & - & $0,30 \pm 0,42$ & - & - & $0,39 \pm 0,17$ & - & - & - & - \\
\hline acetato de etila & - & - & - & - & & - & - & $0,20 \pm 0,12$ & - & $0,24 \pm 0,06$ \\
\hline 2-butenoato de metila & - & - & - & - & - & - & - & $0,20 \pm 0,12$ & - & - \\
\hline butanoato de etila & 802 & & - & - & - & - & - & $2,68 \pm 3,78 \mathrm{a}$ & $7,39 \pm 10,5 \mathrm{a}$ & $0,75 \pm 0,74 \mathrm{a}$ \\
\hline metacrilato de etila & 813 & - & - & - & - & - & - & $4,32 \pm 1,67 \mathrm{a}$ & $5,83 \pm 7,1 \mathrm{a}$ & $3,95 \pm 3,8 \mathrm{a}$ \\
\hline butanoato de propila & 901 & - & - & - & - & - & - & - & $0,53 \pm 0,75$ & - \\
\hline$\alpha$-pineno & 934 & $7,26 \pm 0,23 \mathrm{a}$ & $9,57 \pm 3,09 \mathrm{a}$ & $6,95 \pm 0,20 \mathrm{a}$ & $12,9 \pm 0,12 \mathrm{a}$ & $10,1 \pm 0,67 \mathrm{a}$ & $10,79 \pm 0,12$ & $11,17 \pm 4,91 \mathrm{a}$ & $13,1 \pm 7,88 \mathrm{a}$ & $13,33 \pm 3,06 \mathrm{a}$ \\
\hline não identificado 4 & 947 & - & - & - & - & - & - & - & $0,2 \pm 0,29$ & - \\
\hline canfeno & 951 & - & $0,06 \pm 0,09$ & - & $0,07 \pm 0,10$ & $0,06 \pm 0,09$ & $0,07 \pm 0,10$ & - & $0,2 \pm 0,29$ & $0,09 \pm 0,13$ \\
\hline$\beta$-tujeno & 974 & $0,1 \pm 0,14 \mathrm{a}$ & $0,11 \pm 0,15 \mathrm{a}$ & $0,19 \pm 0,01 \mathrm{a}$ & - & - & - & - & $0,06 \pm 0,08$ & - \\
\hline$\beta$-pineno & 978 & $1,77 \pm 0,06 \mathrm{a}$ & $1,79 \pm 0,01 \mathrm{a}$ & $1,69 \pm 0,06 \mathrm{a}$ & $1,85 \pm 0,33 \mathrm{a}$ & $1,60 \pm 0,25 \mathrm{a}$ & $1,97 \pm 0,32$ & $1,60 \pm 0,96 \mathrm{a}$ & $2,03 \pm 1,48 \mathrm{a}$ & $2,03 \pm 0,35 \mathrm{a}$ \\
\hline$\beta$-mirceno & 990 & $3,98 \pm 0,13 \mathrm{a}$ & $3,94 \pm 0,11 \mathrm{a}$ & $4,17 \pm 0,14 \mathrm{a}$ & $6,86 \pm 2,67 \mathrm{a}$ & $5,43 \pm 3,75 \mathrm{a}$ & $8,72 \pm 1,39$ & $2,71 \pm 0,95 \mathrm{a}$ & $5,14 \pm 5,41 \mathrm{a}$ & $7,24 \pm 1,36 \mathrm{a}$ \\
\hline$\delta$-2-careno & 997 & - & - & - & $0,04 \pm 0,05$ & $0,40 \pm 0,00$ & - & $0,09 \pm 0,0$ & - & - \\
\hline butanoato de butila & 998 & - & - & - & - & - & - & $0,48 \pm 0,67$ & $0,18 \pm 0,26$ & - \\
\hline hexanoato de etila & 1000 & - & - & - & - & - & - & & $1,19 \pm 1,69$ & $0,23 \pm 0,32$ \\
\hline$\delta$-3-careno & 1006 & $60,8 \pm 1,0 \mathrm{a}$ & $57,41 \pm 5,4 \mathrm{a}$ & $59,6 \pm 0,8 \mathrm{a}$ & $33,3 \pm 9,8 \mathrm{a}$ & $42,2 \pm 34,7 \mathrm{a}$ & $27,4 \pm 0,69 \mathrm{a}$ & $47,23 \pm 6,4 \mathrm{a}$ & $33,59 \pm 16,5 \mathrm{a}$ & $25,93 \pm 0,05 \mathrm{a}$ \\
\hline não identificado 5 & 1014 & - & - & - & $0,09 \pm 0,13 \mathrm{a}$ & $0,06 \pm 0,09 \mathrm{a}$ & $0,22 \pm 0,01 \mathrm{a}$ & - & $0,09 \pm 0,13$ & $0,40 \pm 0,08$ \\
\hline$\alpha$-terpineno & 1017 & $1,06 \pm 0,09 \mathrm{a}$ & $1,67 \pm 0,78 \mathrm{a}$ & $0,93 \pm 0,08 \mathrm{a}$ & $2,47 \pm 0,51 \mathrm{a}$ & $1,2 \pm 0,66 \mathrm{a}$ & $2,17 \pm 0,37 \mathrm{a}$ & $1,19 \pm 1,20 \mathrm{a}$ & $1,11 \pm 1,57 \mathrm{a}$ & $2,43 \pm 0,32 \mathrm{a}$ \\
\hline$p$-cimeno & 1025 & $0,46 \pm 0,05 \mathrm{a}$ & $1,21 \pm 1,11 \mathrm{a}$ & $0,53 \pm 0,04 \mathrm{a}$ & $1,93 \pm 0,78 \mathrm{a}$ & $0,74 \pm 0,64 \mathrm{a}$ & $1,4 \pm 0,11 \mathrm{a}$ & $1,03 \pm 1,06 \mathrm{a}$ & $0,67 \pm 0,95 \mathrm{a}$ & $2,06 \pm 0,30 \mathrm{a}$ \\
\hline D-limoneno & 1031 & $6,94 \pm 0,13 \mathrm{a}$ & $6,77 \pm 0,04 \mathrm{a}$ & $7,14 \pm 0,15 \mathrm{a}$ & $9,29 \pm 2,56 \mathrm{a}$ & $6,52 \pm 3,00 \mathrm{a}$ & $9,77 \pm 1,64 \mathrm{a}$ & $4,6 \pm 2,22 \mathrm{a}$ & $6,95 \pm 6,38 \mathrm{a}$ & $9,63 \pm 1,29 \mathrm{a}$ \\
\hline$(Z)$ - $\beta$-ocimeno & 1040 & - & - & - & $0,03 \pm 0,04 \mathrm{a}$ & $0,24 \pm 0,08 \mathrm{a}$ & $0,31 \pm 0,43 \mathrm{a}$ & - & - & $0,1 \pm 0,14$ \\
\hline$(E)$ - $\beta$-ocimeno & 1050 & $0,04 \pm 0,05 \mathrm{a}$ & $0,08 \pm 0,12 \mathrm{a}$ & $0,05 \pm 0,08 \mathrm{a}$ & $0,26 \pm 0,37 \mathrm{a}$ & $0,5 \pm 0,09 \mathrm{a}$ & $0,32 \pm 0,24 \mathrm{a}$ & - & $0,17 \pm 0,24$ & $0,21 \pm 0,29$ \\
\hline$\beta$-felandreno & 1056 & $0,04 \pm 0,06 \mathrm{a}$ & $0,02 \pm 0,03 \mathrm{a}$ & $0,07 \pm 0,02 \mathrm{a}$ & $0,07 \pm 0,11$ & $0,05 \pm 0,08$ & - & - & $0,05 \pm 0,08$ & $0,1 \pm 0,14$ \\
\hline$\gamma$-terpineno & 1062 & $0,81 \pm 0,01 \mathrm{a}$ & $0,70 \pm 0,13 \mathrm{a}$ & $0,84 \pm 0,01 \mathrm{a}$ & $1,1 \pm 0,18 \mathrm{a}$ & $0,6 \pm 0,85 \mathrm{a}$ & $1,33 \pm 0,22 \mathrm{a}$ & $0,72 \pm 0,25 \mathrm{a}$ & $1,11 \pm 0,57 \mathrm{a}$ & $1,17 \pm 0,03 \mathrm{a}$ \\
\hline butanoato de isoamila & 1064 & - & - & - & - & - & - & $0,21 \pm 0,29$ & $0,33 \pm 0,47$ & - \\
\hline$\alpha$-terpinoleno & 1089 & $8,66 \pm 0,38 \mathrm{a}$ & $7,59 \pm 1,06 \mathrm{a}$ & $9,22 \pm 0,41 \mathrm{a}$ & $13,1 \pm 6,6 \mathrm{a}$ & $9,56 \pm 6,99 \mathrm{a}$ & $13,6 \pm 5,13 \mathrm{a}$ & $4,58 \pm 1,65 \mathrm{a}$ & $8,83 \pm 9,25 \mathrm{a}$ & $12,88 \pm 3,72 \mathrm{a}$ \\
\hline$p$-menta-1,5-dien-8-ol & 1167 & - & - & - & - & $0,04 \pm 0,05$ & - & & - & - \\
\hline limonen-4-ol & 1176 & - & - & - & $0,15 \pm 0,22 \mathrm{a}$ & $0,19 \pm 0,27 \mathrm{a}$ & $0,2 \pm 0,20 \mathrm{a}$ & $0,18 \pm 0,00$ & - & - \\
\hline isomentol & 1182 & - & - & - & - & $0,13 \pm 0,19$ & - & $0,09 \pm 0,00$ & - & - \\
\hline 1-terpinen-4-ol & 1185 & $0,46 \pm 0,06 \mathrm{a}$ & $0,25 \pm 0,36 \mathrm{a}$ & $0,37 \pm 0,06 \mathrm{a}$ & - & - & $0,14 \pm 0,0$ & - & - & - \\
\hline$p$-cimen-8-ol & 1191 & - & - & - & - & $0,12 \pm 0,17$ & $0,16 \pm 0,23$ & $0,20 \pm 0,00$ & - & - \\
\hline octanoato de etila & 1196 & - & - & - & - & - & - & - & - & $0,08 \pm 0,11$ \\
\hline$\alpha$-terpineol & 1198 & $0,27 \pm 0,05 \mathrm{a}$ & $0,15 \pm 0,21 \mathrm{a}$ & $0,24 \pm 0,05 \mathrm{a}$ & - & - & - & - & - & - \\
\hline eucarvona & 1251 & $0,3 \pm 0,01 \mathrm{a}$ & $0,15 \pm 0,22 \mathrm{a}$ & $0,27 \pm 0,02 \mathrm{a}$ & - & $0,09 \pm 0,13$ & - & - & - & - \\
\hline$(E)$-anetol & 1291 & - & - & - & - & $0,09 \pm 0,13$ & - & - & - & - \\
\hline$\alpha$-cubebeno & 1351 & - & - & - & - & $0,07 \pm 0,09$ & - & - & - & - \\
\hline$\alpha$-copaeno & 1383 & $0,03 \pm 0,04 \mathrm{a}$ & $0,33 \pm 0,43 \mathrm{a}$ & $0,07 \pm 0,01 \mathrm{a}$ & $2,37 \pm 1,03 \mathrm{a}$ & $1,78 \pm 0,76 \mathrm{a}$ & $0,97 \pm 1,11 \mathrm{a}$ & $1,43 \pm 0,81 \mathrm{a}$ & $1,08 \pm 0,01 \mathrm{a}$ & $2,66 \pm 0,70 \mathrm{a}$ \\
\hline$\alpha$-gurjuneno & 1415 & $0,47 \pm 0,01 \mathrm{a}$ & $0,42 \pm 0,06 \mathrm{a}$ & $0,49 \pm 0,01 \mathrm{a}$ & $0,86 \pm 0,42 \mathrm{a}$ & $0,91 \pm 0,80 \mathrm{a}$ & $0,87 \pm 0,41 \mathrm{a}$ & $0,97 \pm 0,03 \mathrm{a}$ & $0,63 \pm 0,25 \mathrm{a}$ & $0,72 \pm 0,10 \mathrm{a}$ \\
\hline trans- $\beta$-cariofileno & 1428 & $2,26 \pm 0,28 \mathrm{a}$ & $2,30 \pm 0,35 \mathrm{a}$ & $2,66 \pm 0,28 \mathrm{a}$ & $5,98 \pm 0,73 a$ & $9,04 \pm 9,96 \mathrm{a}$ & $10,31 \pm 4,50 \mathrm{a}$ & $4,76 \pm 2,09 \mathrm{a}$ & $4,64 \pm 1,35 \mathrm{a}$ & $5,76 \pm 1,06 \mathrm{a}$ \\
\hline$\alpha$-cariofileno & 1464 & $1,13 \pm 0,17 \mathrm{a}$ & $1,03 \pm 0,04 \mathrm{a}$ & $1,36 \pm 0,15 \mathrm{a}$ & $2,63 \pm 0,52 \mathrm{a}$ & $4,3 \pm 4,89 \mathrm{a}$ & $5,49 \pm 2,31 \mathrm{a}$ & $2,04 \pm 0,90 \mathrm{a}$ & $1,98 \pm 0,61 \mathrm{a}$ & $2,58 \pm 0,51 \mathrm{a}$ \\
\hline$\gamma$-elemeno & 1469 & - & - & - & - & - & $0,21 \pm 0,29$ & - & - & - \\
\hline$\alpha$-amorfeno & 1478 & - & - & - & - & - & $0,12 \pm 0,17$ & - & - & - \\
\hline germacreno D & 1489 & - & - & - & - & $0,15 \pm 0,21$ & $0,54 \pm 0,76$ & - & - & - \\
\hline$\beta$-selineno & 1498 & $0,64 \pm 0,13 \mathrm{a}$ & $0,59 \pm 0,07 \mathrm{a}$ & $0,82 \pm 0,13$ & $1,46 \pm 0,65 \mathrm{a}$ & $0,85 \pm 1,20 \mathrm{a}$ & $1,38 \pm 0,54 \mathrm{a}$ & $1,60 \pm 0,10 \mathrm{a}$ & $0,94 \pm 0,03 b$ & $1,40 \pm 0,17 \mathrm{ab}$ \\
\hline ledeno & 1504 & - & - & - & $0,1 \pm 0,14 \mathrm{a}$ & $0,12 \pm 0,17 \mathrm{a}$ & $0,55 \pm 0,42 \mathrm{a}$ & - & - & - \\
\hline monoterpenos & & $93,02 \pm 0,52$ & $91,47 \pm 2,72$ & $92,32 \pm 0,46$ & $83,44 \pm 1,76$ & $79,91 \pm 16,15$ & $78,30 \pm 9,50$ & $75,37 \pm 6,5$ & $73,3 \pm 18,4$ & $77,22 \pm 3,71$ \\
\hline sesquiterpenos & & $4,53 \pm 0,64$ & $4,67 \pm 0,83$ & $5,39 \pm 0,58$ & $13,39 \pm 0,71$ & $17,19 \pm 18,01$ & $18,90 \pm 10,1$ & $10,79 \pm 3,93$ & $9,26 \pm 1,67$ & $13,11 \pm 2,54$ \\
\hline ésteres & & - & - & - & - & - & - & $8,08 \pm 3,07$ & $15,46 \pm 20,7$ & $5,23 \pm 5,14$ \\
\hline Total de compostos & & 22 & 25 & 22 & 25 & 33 & 26 & 27 & 29 & 27 \\
\hline
\end{tabular}

a Área relativa do pico no cromatograma obtido por CG-EM. Em cada estádio de maturação, médias seguidas de letra igual, na horizontal, não diferem significativamente pelo teste de Tukey a $5 \%$ de probabilidade. 
Tabela 2. Parâmetros de desempenho analítico do método cromatográfico desenvolvido para determinação de mangiferina em polpa de manga: linearidade (r), precisão intradia e interdia $(\mathrm{CV})$, exatidão $\mathrm{C}_{\mathrm{e}} / \mathrm{C}_{\mathrm{t}}$

\begin{tabular}{|c|c|c|c|c|c|c|c|c|c|c|}
\hline \multirow{2}{*}{$\begin{array}{l}\text { mangiferina } \\
(\mu \mathrm{g} / \mathrm{mL})\end{array}$} & \multicolumn{3}{|c|}{$\begin{array}{c}1^{\circ} \text { dia } \\
y=80006 x-88287\end{array}$} & \multicolumn{3}{|c|}{$\begin{array}{c}2^{\circ} \text { dia } \\
y=89080 x-413469\end{array}$} & \multicolumn{3}{|c|}{$\begin{array}{c}3^{\circ} \text { dia } \\
y=82591 x-106876\end{array}$} & \multirow{2}{*}{$\begin{array}{l}\text { Precisão } \\
\text { interdia } \\
\mathrm{CV}(\%)\end{array}$} \\
\hline & $\mathrm{CV}^{\mathrm{a}}(\%)$ & $\mathrm{C}_{\mathrm{e}} / \mathrm{C}_{\mathrm{t}}^{\mathrm{b}}(\%)$ & $\mathrm{r}^{\mathrm{c}}$ & $\mathrm{CV}(\%)$ & $\mathrm{C}_{\mathrm{e}} / \mathrm{C}_{\mathrm{t}}(\%)$ & $\mathrm{r}$ & $\mathrm{CV}(\%)$ & $\mathrm{C}_{\mathrm{e}} / \mathrm{C}_{\mathrm{t}}(\%)$ & $\mathrm{r}$ & \\
\hline 20 & 1,65 & 105,0 & 0,9995 & 1,11 & 117,5 & 0,9992 & 1,01 & 107,5 & 0,9995 & 1,90 \\
\hline 130 & 0,99 & 101,7 & & 5,08 & 103,5 & & 1,44 & 104,7 & & 2,50 \\
\hline 250 & 0,20 & 100,9 & & 1,57 & 98,6 & & 2,18 & 100,7 & & 1,32 \\
\hline
\end{tabular}

${ }^{a}$ Coeficiente de variação. ${ }^{\text {b }}$ Razão entre concentração determinada experimentalmente/concentração teórica. ${ }^{\mathrm{c}}$ Coeficiente de correlação das curvas de calibração.

de mangiferina considerados como verdadeiros, teóricos $\left(\mathrm{C}_{\mathrm{t}}\right)$, em 3 níveis de concentração $(20,130$ e $250 \mu \mathrm{g} / \mathrm{mL})$. A exatidão foi maior nas determinações efetuadas nas soluções mais concentradas $(98,6$ $100,9 \%)$ e menor nas soluções mais diluídas (105,0-117,5\%). As determinações de mangiferina mostraram-se exatas, tendo em vista que em apenas uma análise o grau de concordância entre a concentração prática e teórica foi superior ao limite aceitável $\left(\mathrm{C}_{\mathrm{e}} / \mathrm{C}_{\mathrm{t}}>115 \%\right){ }^{21}$

$\mathrm{O}$ método validado foi adotado na quantificação de mangiferina em frutos submetidos a tratamentos com 1-MCP e AVG, em três estádios de maturação. Os teores de mangiferina (expressos em mg/ $\mathrm{kg}$ de polpa fresca) foram determinados, em triplicata, em amostras tratadas e não tratadas com inibidores de etileno, à medida que os frutos foram amadurecendo (Tabela 3 ). Nos frutos do grupo controle, os teores de mangiferina variaram de $0,33-2,11 \mathrm{mg} / \mathrm{kg}$, dependendo do estádio de maturação. Considerando o teor de umidade de cerca de $80 \%$ desta variedade, ${ }^{22}$ estes valores foram compatíveis com o teor determinado por Berardinini e colaboradores através de CLAE (4,6 mg/kg na polpa seca). ${ }^{23}$ Entretanto, este método apresenta como grande inconveniente a necessidade de liofilização da amostra, que o torna mais dispendioso e menos prático do que o método desenvolvido neste trabalho.

Tabela 3. Concentração de mangiferina em manga 'Tommy Atkins' tratada e não tratada com inibidores de etileno, em diferentes estádios de maturação

\begin{tabular}{cccc}
\hline \multirow{2}{*}{$\begin{array}{c}\text { Manga } \\
\text { Estádio de maturação }\end{array}$} & \multicolumn{3}{c}{$\begin{array}{c}\text { mangiferina } \\
(\mathrm{mg} / \mathrm{kg} \text { de polpa fresca })\end{array}$} \\
\hline 2 & $1,63 \pm 0,33 \mathrm{a}$ & $1,75 \pm 0,18 \mathrm{a}$ & $2,11 \pm 0,31 \mathrm{a}$ \\
3 & $1,01 \pm 0,06 \mathrm{a}$ & $1,47 \pm 0,30 \mathrm{a}$ & $0,33 \pm 0,29 \mathrm{~b}$ \\
4 & $0,76 \pm, 72 \mathrm{a}$ & $1,12 \pm 0,24 \mathrm{a}$ & $1,27 \pm 0,79 \mathrm{a}$ \\
\hline
\end{tabular}

${ }^{a}$ Médias seguidas de letra igual, na horizontal, não diferem significativamente pelo teste de Tukey a $5 \%$ de probabilidade.

Em frutos avaliados no estádio de maturação 2, os teores de mangiferina não foram estatisticamente diferentes (AVG- 1,63; 1-MCP- 1,75 e controle- $2,11 \mathrm{mg} / \mathrm{kg}$ ). No entanto, na manga no estádio de maturação 3 , a concentração em frutos não tratados foi significativamente menor $(0,33 \mathrm{mg} / \mathrm{kg})$, indicando uma suposta interferência dos inibidores de etileno na biossíntese de mangiferina. No estádio final de maturação, os níveis de concentração voltaram a ser relativamente similares (AVG- 0,76; 1-MCP- 1,12 e controle$1,27 \mathrm{mg} / \mathrm{kg}$ ) e sinalizaram uma tendência de declínio na produção de mangiferina ao longo do amadurecimento, tal como já observado para o conteúdo total de compostos fenólicos na cultivar Alfonso. ${ }^{24}$

\section{CONCLUSÃO}

Análises cromatográficas por CG-EM e CLAE revelaram que não há diferenças significativas nas concentrações dos componentes voláteis e de mangiferina em mangas 'Tommy Atkins' tratadas com 1-MCP e AVG e que, portanto, o uso de inibidores de etileno não afeta o aroma e o potencial nutricional/funcional (em termos de mangiferina) da fruta, duas propriedades consideradas importantes pelo consumidor na avaliação de qualquer alimento.

\section{AGRADECIMENTOS}

À Embrapa/BID pelo suporte financeiro concedido (AGROFUTURO- 03.07.05.034.00.00), ao Prof. E. R. Silveira (CENAUREMNUFC) pela cortesia em disponibilizar suas facilidades laboratoriais, e ao Prof. A. M. E. Bezerra (UFC) pela orientação prestada na análise estatística.

\section{REFERÊNCIAS}

1. Taiz, L.; Zeiger, E.; Fisiologia vegetal, $3^{\mathrm{a}}$ ed., Artmed: Porto Alegre, 2004; Wang, C. Y.; Acta Hort. 1987, 201,187.

2. Sisler, E. C.; Biotechnol. Adv. 2006, 24, 357; Watkins, C. B.; Biotechnol. Adv. 2006, 24, 389.

3. Blankenship, S. M.; Dole, J. M.; Postharvest Biol. Technol. 2003, $28,1$.

4. Plutowska, B.; Wardencki, W.; Food Chem. 2007, 101, 845; Espin, J. C.; Garcia-Conesa, M. T.; Tomás-Barberán, F. A.; Phytochemistry 2007, 68, 2986.

5. Kondo, S.; Setha, S.; Rudell, D. R.; Buchanan, D. A.; Mattheis, J. P.; Postharvest Biol. Technol. 2005, 36, 61; Fan, X.; Mattheis, J. P.; Buchanan, D.; J. Agric. Food. Chem 1998, 46, 1959; Moya-León; M. A.; Vergara, M.; Bravo, C.; Montes, M. E.; Moggia, C.; Postharvest Biol. Technol. 2006, 42, 185; Argenta, L. C.; Fan, X.; Mattheis, J. P.; J. Agric. Food. Chem. 2003, 51, 3858.

6. do Nascimento Júnior, B. B.; Rezende, C. M.; Soares, A. G.; Fonseca, M. J. de O.; Quim. Nova 2008, 31, 1367.

7. Lurie, S.; Pre-Aymard, C.; Ravid, U.; Larkov, O.; Fallik, E.; J. Agric. Food. Chem. 2002, 50, 4251; Marin, A. B.; Colonna, A. E.; Kudo, K.; Kupferman, E. M.; Mattheis, J. P.; Postharvest Biol. Technol. 2009, 51, 73.

8. Rizzolo, A.; Cambiaghi, P.; Grassi, M.; Zerbini, P. E.; J. Agric. Food. Chem. 2005, 53, 9781.

9. Flores, F.; El-yahyaoul, E.; Billerbeck, G.; Romojaro, F.; Latché, A.; Bouzayen, M.; Pech, J. C.; Ambid, C.; J. Exp. Bot. 2002, 53, 201; Golding, J. B.; Shearer, D.; Mcglasson, W. B.; Willyie, S. G.; J. Agric. Food Chem. 1999, 47, 1646.

10. Lalel, H. J. D.; Singh, Z.; Tan, S. C.; J. Hort. Sci. Biotechnol. 2003, 78, 131.

11. Wang, B.; Wang, J.; Feng, X.; Lin, L.; Zhao, Y.; Jiang, W.; Plant Growth Regul. 2009, 57, 185.

12. Mozetic, B.; Simcic, M.; Tresbe, P.; Food Chem. 2006, 97, 302.; MacLean, D. D.; Murr, D. P.; Deell, J.; Horvath, C.; J. Agric. Food. Chem. 2006, 54, 870; MacLean, D. D.; Murr, D. P.; Deell, J. R.; Mackay, A. B.; Kupferman, E. M.; Postharvest Biol. Technol. 2007, 45, 46.

13. Pinto, A. C. Q. Em Agricultura Tropical: quatro décadas de inovações tecnológicas, institucionais e políticas; Albuquerque, A. C. S.; Silva, A. G., eds.; Embrapa Informação Tecnológica: Brasília, 2008, cap. 20. 
14. Lima, M. A. C; Silva, A. L.; Azevedo, S. S. N.; Santos, P. S.; Rev. Bras. Frutic. 2006, 28, 64; Santos, A. C. N.; Lima, M. A. C.; Trindade. D. C. G.; Ribeiro, T. P.; Souza, S. O.; Costa, A. C. S., Amariz, A.; Embrapa Semiárido - Documentos 2006, 197, 225.

15. Canuto, K. M.; Souza-Neto, M. A.; Garruti, D. S.; Quim. Nova 2009, 32, 2377; Lopes, D. C.; Fraga, S. R.; Rezende, C. M.; Quim. Nova 1999, 22, 31.

16. Barreto, J. C.; Trevisan, M. T. S.; Hull, W. E.; Erben, G.; De Brito, E. S.; Pfundstein, B.; Würtele, G.; Spiegelhalder, B.; Owen, R. W.; J. Agric. Food. Chem. 2008, 56, 5599.

17. Andreu, G. P.; Delgado, R.; Velho, J. A.; Curti, C.; Vercesi, A. E.; Eur. J. Pharmacol. 2005, 513, 47; Rodriguez, J.; Di Piero, D.; Gioia, M.; Monaco, S.; Delgado, R.; Coletta, M.; Marini, S.; Biochim. Biophys. Acta 2006, 1760, 1333; Leiro, J.; Arranz, J. A.; Yáñez, M.; Ubeira, F. M.; San-Martin, M. L.; Orallo, F.; Int. Immunopharmacol. 2004, 4, 763; Huang, T. H-W.; Peng, G.; Li, G. Q.; Yamahara, J.; Roufogalis, B. D.; Li; Y.; Toxicol. Appl. Pharmacol. 2006, 210, 225; Yoshimi, N.; Matsunaga, K.; Katayama, M.; Yamada, Y.; Kuno, T.; Qiao, Z.; Hara, A.; Yamahara, J.; Mori, H.; Cancer Lett. 2001, 163, 163; Rajendran, P.; Ekambaram, G.; Sakthisekaban, D.; Biol. Pharm. Bull. 2008, 31, 1053; Carvalho, A. C. S.; Souza, A. L.; Guedes, M. M.; Trevisan, M.
T. S.; Lima, A. F.; Santos, F. A.; Rao, V. S. N.; Planta Med. 2007, 73, 1372; Núñez-Sellés, A. J.; Delgado-Hernández, R.; Garrido-Garrido, G.; García-Rivera, D.; Guevara-García, M.; Pardo-Andreu, G. L.; Pharmacol. Res. 2007, 55, 351; Garrido, G.; González, D.; Romay, C.; Núñez-Sellés, A. J.; Delgado, R.; Food Chem. 2008, 107, 1008; NúñezSellés, A. J.; J. Braz. Chem. Soc. 2005, 16, 699.

18. http://webbook.nist.gov/chemistry/, acessada em Junho 2009 e Junho 2010.

19. Defilippi, B. G.; Kader, A. A.; Dandekar, A. M.; Plant Sci. 2005, 168, 1189.

20. Botondi, R.; Desantis, D.; Bellincontro, A.; Vizovitis, K.; Mencarelli, F.; J. Agric. Food Chem. 2003, 51, 1189.

21. Agência Nacional de Vigilância Sanitária (ANVISA); Resolução $n^{o} R E$ 899, de 29 de maio de 2003.

22. Rodrigues, L. K.; Pereira, L. M.; Ferrari, C. C.; Sarantópulos, C. I. G. L.; Hubinger, M. D.; Ciênc. Tecnol. Aliment. 2008, 28, 271.

23. Berardini, N.; Fezer, R.; Conrad, J.; Beifuss, U.; Carle, R.; Schieber, A.; J. Agric. Food Chem. 2005, 53, 1563.

24. Lakshminarayana, S.; Subhadra, N. V.; Subramanyam, H.; J. Hort. Sci. 1970, 45, 133. 